\title{
Research on Evaluation of the Comprehensive Benefit of Power Grid Value-added Service Based on Grey Whitening Weight Function
}

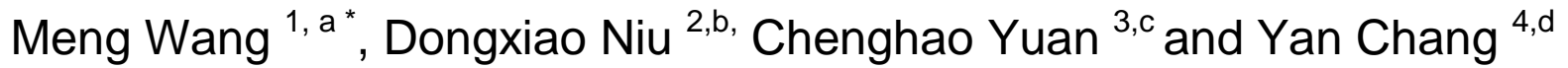 \\ ${ }^{1-3}$ School of Economic and Management, North China Electric Power University, Changping District, \\ Beijing 102206, China; \\ ${ }^{4}$ State Grid Energy Research Institute, Changping District, Beijing 102209, China \\ awmeng3007@163.com, bniudx@126.com, cychncepu@163.com, demily_chang@126.com \\ *The Corresponding Author
}

Keywords: Value added service of power grid enterprise; Grey whitening weight function; Comprehensive benefit; Evaluation

\begin{abstract}
The power grid enterprises are facing a new competitive situation under the new electricity reform, and the demand for value-added service is becoming more and more vigorous. How to evaluate the comprehensive benefit of the value-added service of the power grid enterprise through scientific calculation is crucial to the decision and improvement of the enterprise. The index system of benefit evaluation is constructed according to the business characteristics, and an evaluation model based on Grey whitening weight function is put forward. And take "e charging" as an example to carry out the empirical analysis which can provide reference for other business.
\end{abstract}

\section{Introduction}

As the result of the development of big data and the Increasingly competitive environment, major industries have launched many value-added services and tried to improve them. Under the reformation of electric power system, the task of improving the quality of operations and management, improving service levels, diversifying service businesses, developing new markets, and nurturing new businesses is very urgent for power grid enterprise.It is the next important step for power grid enterprise to comprehensively carry out value-added service businesses. In this article, we set up the indicator system and the construction model for grid enterprise data value-added services so as to provide decision support for the company to evaluate the potential of value-added services, whether to promote value-added services and provide an evaluation basis for the company to find out the short boards and problems of value-added services that have been launched.

At present, the research on the construction and evaluation model of value-added service indicator system is not perfect enough. Considering the similarity between smart grid and smart grid, we can refer to the related research of smart grid to set up the indicator system. I developed a set of power customer segmentation and value-added service system for power supply enterprises, using new algorithm to analyze the customers in this area, forming different characteristic customer groups, and customizing personalized value-added service menu according to different needs characteristics. Reference Literature [1] develops a set of power customer segmentation and value-added service systems for power supply companies, using new algorithms to analyze customers in Maoming to form customer groups with different characteristics, and customizes personalized value-added services for customer groups according to different demand characteristics. Reference Literature [2,3] takes the comprehensive value of electricity customers as the assessment direction, the electricity customer's electricity consumption indicators as the evaluation content to construct the customer's comprehensive value evaluation method, and the obtained subdivided results provides auxiliary support for the power supply bureau to formulate a personalized value-added service strategy. In order to ensure the standardized development of the power value-added service system for the diversified power market, Reference Literature [4] carried 
out a demand analysis of the power value-added service platform for diversified power markets, and proposed seven major functional areas to further introduce 11 functional modules, business processes and interface descriptions. Reference Literature [5] establishes an evaluation indicator system based on corporate ecology theory. It mainly considers the three dimensions of external environment, corporate profitability, and social contribution. Delphi method and AHP are used. Reference Literature [6,7] constructs the indicator system from the four aspects of environmental benefits, economic benefits, social benefits and safety, and uses the AHP method to evaluate the value-added service benefits of the grid. Reference Literature [8] which starts from the three links of power generation, transmission and electricity use considers the external environment, profitability, social contribution, market conditions, business foundation, and talent skills to build an evaluation indicator system of smart grid and build an entropy weight-AHP evaluation model. Reference Literature [9] mainly considers the development level indicator system and the effectiveness indicator system. The level indicator system is used to describe the degree of development of key technologies in power generation, transmission, substation, power distribution, electricity use, and dispatch. The effectiveness indicator system reflects the benefits and impact that the power grid construction can bring to power generation companies, power grid companies, power users, and the society. The evaluation indicator of grid value-added services constructed in Reference Literature [10] includes project technical indicators, economic indicators, social indicators, practical indicators, project management indicators, and innovative indicators.

\section{Comprehensive Benefit Evaluation Model Based on Grey System Whitening Weight Function}

The Indicators System of Comprehensive Benefit. For power grid enterprise, the indicators for evaluating value-added services should not only be based on financial indicators, but also need to consider the social and environmental benefits it brings. In addition, due to the variety of value-added services, it is necessary to integrate various types of services in order to determine evaluation indicators. From the three aspects of economic benefits, social benefits and environmental benefits, with the various value-added service application scenarios comprehensively considered, we can get the evaluation system of the value-added service for power grid enterprise. The details are shown in Table 1.

Table 1 Indicator System of Benefits Evaluation for Grid Enterprises' Value-added Services

\begin{tabular}{|c|c|c|}
\hline $\begin{array}{l}\text { First grade } \\
\text { indicatores }\end{array}$ & Second grade indicatores & Principles of indicator selection \\
\hline \multirow{3}{*}{$\begin{array}{l}\text { Economic benefit } \\
\text { A }\end{array}$} & Profit level $\mathrm{A}_{1}$ & $\begin{array}{l}\text { Measurement of profitability of value-added services, } \\
\text { the core of financial analysis. }\end{array}$ \\
\hline & Operation level $\mathrm{A}_{2}$ & $\begin{array}{c}\text { Analysis of business operations helps improve the level } \\
\text { and efficiency of management. }\end{array}$ \\
\hline & Development potential $A_{3}$ & $\begin{array}{l}\text { Reflection of the market potential of value-added } \\
\text { services, such as the increase in market share, etc. }\end{array}$ \\
\hline \multirow{3}{*}{ Social benefitsB } & Satisfaction of related party & $\begin{array}{l}\text { Customer satisfaction, which is the match between } \\
\text { customer expectations and customer experience. }\end{array}$ \\
\hline & $\begin{array}{l}\text { Promoting of regional economic } \\
\text { development } \mathrm{B}_{2}\end{array}$ & $\begin{array}{c}\text { Impact on the regional economy, whether it can directly } \\
\text { or indirectly promote the economic development of } \\
\text { related regions. }\end{array}$ \\
\hline & $\begin{array}{c}\text { Promotion of technology } \\
\text { development } \mathrm{B}_{3}\end{array}$ & $\begin{array}{l}\text { Promotion of the society's existing technology, whether } \\
\text { it can promote scientific and technological progress. }\end{array}$ \\
\hline \multirow{2}{*}{$\begin{array}{l}\text { Environmental } \\
\text { benefits } \mathrm{C}\end{array}$} & Resource savings $\mathrm{C}_{2}$ & $\begin{array}{c}\text { The degree of conservation of various resources due to } \\
\text { the development of value-added services, whether it can } \\
\text { promote ecological protection. }\end{array}$ \\
\hline & $\begin{array}{l}\text { Reduction of environmental } \\
\text { pollution } \mathrm{C}_{3}\end{array}$ & $\begin{array}{c}\text { Whether contributes to reduce environmental pollution } \\
\text { such as water pollution, solid waste pollution, air } \\
\text { pollution, and thermal pollution }\end{array}$ \\
\hline
\end{tabular}


Evaluation Model. The value-added services of power grid enterprise have the characteristics of a gray system. System factors are not completely clear and the factors are not completely clear. The principal component analysis method, fuzzy comprehensive evaluation method and multi-indicator comprehensive evaluation method based on neural network need a lot of evaluation object data to be able to perform better. The whitening weight function, which is one of the theoretical pillars of the grey comprehensive evaluation method, is based on known information and is a subjective judgement of the degree of "preference" of a gray number or grey class within its range of values. The method describes how well each data point is affiliated with the gray number or gray class, and is more suitable for value-added services of power grid enterprise. Therefore, this paper constructs a comprehensive evaluation method based on gray system whitening weight function to evaluate.

The method for benefit evaluation using the gray whitening weight function method is as follows:

\section{Evaluation Factor Sets and indicator Weight Sets}

Hypothesis $U=\left\{u_{1}, u_{2}, \cdots, u_{p}\right\}$ is a set of evaluation factors consisting of $p$ types of factors, and the overall goal of evaluation is $U$. Under the factor ${ }^{i}, n_{i}$ evaluation indicator $\mathrm{A}$ are set $v_{i j}\left(j=1,2, \cdots, n_{i}\right)$. Different evaluation indexes have different influences on the evaluation of the total goals $U$, so the AHP method is used to determine the weight of each indicator. Hypothesis $\alpha=\left(\alpha_{1}, \alpha_{2}, \cdots, \alpha_{p}\right)$ as the weight vector relative to $U$, and the number of factors is $p$.Hypothesis the weight vector of the $p$ evaluation indicators $v_{i j}$ relative to $U_{i}$ under the $i$ factor as $\omega_{i}=\left(\omega_{i 1}, \omega_{i 2}, \cdots, \omega_{i p}\right)$

\section{Calculate the Sample Matrix of the Evaluation Indicators}

Assume that $\mathrm{m}$ experts are invited to evaluate the observations of $n$ economic benefit indicators of an evaluation object. The $k(k=1,2, \cdots, \mathrm{m})$ expert gives the score of the observed value of the $i$ indicator as $b_{i}^{k}(k=1,2, \cdots, \mathrm{m})$, and thus the sample matrix of evaluation indicators $B$ is:

$$
B=\left[\begin{array}{cccc}
b_{1}{ }^{1} & b_{1}{ }^{2} & \cdots & b_{1}{ }^{m} \\
b_{2}{ }^{1} & b_{2}{ }^{2} & \cdots & b_{2}{ }^{m} \\
\cdots & \cdots & \cdots & \cdots \\
b_{n}{ }^{1} & b_{n}{ }^{2} & \cdots & b_{n}{ }^{m}
\end{array}\right]=\left(b_{i}^{k}\right)_{n \times m}
$$

\section{Determine Evaluation Gray Class and its Whitening Weight Function}

The $\mathrm{n} \times \mathrm{m}$ scores given by $m$ experts are called whitening values. To determine the gray class to which each indicator belongs based on the whitening value, first we need to set the number of gray classes, the range of values for each gray class, and the whitening weight function for each gray class. Assume that $M$ gray classes are divided, and the value range $\left[b_{1}, b_{M+1}\right]$ of the $i$ indicator is divided as $\left[b_{1}, b_{2}\right], \ldots\left[b_{k}, b_{k+1}\right], \ldots,\left[b_{M}, b_{M+1}\right]$. Assume the $N$ whitening weight functions of the $i$ indicator as $f_{i}^{N}(x)$.The commonly used whitening weight function consists of the upper limit measurement whitening weight function, the moderate measurement whitening weight function, and the lower-level whitening weight function.

When its gray number is $\otimes \in\left[b_{i}^{N}(1), b_{i}^{N}(2),-,-\right]$, it is called upper limit measurement whitening weight function. Its function expression is as follows:

$$
f_{i}^{N}(x)=\left\{\begin{array}{l}
0, x<b_{i}^{N}(1) \\
\frac{x-b_{i}^{N}(1)}{b_{i}^{N}(2)-b_{i}^{N}(1)} \\
1, x>b_{i}^{N}(2)
\end{array}, x \in\left[b_{i}^{N}(1), b_{i}^{N}(2)\right]\right.
$$

In the above function, $b_{i}^{N}(\bullet)$ is the threshold value of gray class $N$, and $x$ is the observed value of the $i$ index. 
When its gray number is $\otimes \in\left[b_{i}^{N}(1), b_{i}^{N}(2),-, b_{i}^{N}(4)\right]$, it is called moderate measurement whitening weight function. Its function expression is as follows:

$$
f_{i}^{N}(x)=\left\{\begin{array}{cc}
0 & x \notin\left[b_{i}^{N}(1), b_{i}^{N}(4)\right] \\
\frac{x-b_{i}^{N}(1)}{b_{i}^{N}(2)-b_{i}^{N}(1)} & x \in\left[b_{i}^{N}(1), b_{i}^{N}(2)\right] \\
\frac{b_{i}^{N}(4)-x}{b_{i}^{N}(4)-b_{i}^{N}(2)} & x \in\left[b_{i}^{N}(2), b_{i}^{N}(4)\right]
\end{array}\right.
$$

When its gray number is $\otimes \in\left[-,-, b_{i}^{N}(3), b_{i}^{N}(4)\right]$, it is called lower limit measurement whitening weight function. Its function expression is as follows:

$$
f_{i}^{N}(x)=\left\{\begin{array}{cc} 
& \\
0 & x \notin\left[0, b_{i}^{N}(4)\right] \\
1 & x \in\left[0, b_{i}^{N}(3)\right] \\
\frac{b_{i}^{N}(4)-x}{b_{i}^{N}(4)-b_{i}^{N}(3)} & x \in\left[b_{i}^{N}(3), b_{i}^{N}(4)\right]
\end{array}\right.
$$

Calculate the coefficient and weight of gray evaluation

For the $i$ indicator, the gray evaluation system of the $N$ gray class is ${ }^{x_{i, N}}$ :

$$
x_{i, N}=\sum_{k=1}^{m} f_{N}\left(b_{i}^{k}\right)
$$

The total gray evaluation coefficient for all types of evaluation gray is $x_{i}$ :

$$
x_{i}=\sum_{N=1}^{M} x_{i, N}
$$

Gray evaluation weights belonging to the $N$ gray class are marked as $r_{i, N}$ :

$$
r_{i, N}=\frac{x_{i, N}}{x_{i}}
$$

The gray class weight vector for the $i$ indicator is $R_{i, N}$ :

$$
R_{i, N}=\left(r_{i, 1}, r_{i, 2}, \cdots, r_{i, N}\right)
$$

Grey comprehensive evaluation

The comprehensivel clustering matrix for an evaluation object for the $N$ gray class is:

$$
R_{N}=W \times\left(R_{1, N}, R_{2, N}, \cdots, R_{n, N}\right)
$$

Among them, $R_{i, N}=(i=1,2, \cdots, n)$ is the gray class weight vector for the $i$ indicator.

For $\max \left\{R_{N}\right\}=R_{N^{*}}, 1 \leq N \leq M$, it can be judged that the evaluation object belongs to gray class $N^{*}$.

\section{Empirical Analysis}

The "e-charging" vehicle networking platform is a vehicle networking intelligent platform developed and operated by the State Grid Corporation of China, which integrates charging and replacing facilities monitoring, information services, tariff settlement, vehicle networking services, and life services. Through the vehicle networking platform, the business of charging, switching, recharging, pinning, and reporting of electric vehicle charging cards can be completed easily, which greatly meets the charging requirements of the majority of electric vehicle owners. As the most 
intelligent and convenient mobile phone client in China, the number of "e-charging" service users accounts for $50 \%$ of the 600,000 passenger cars in China. Taking "e charging" as an example, we evaluate the comprehensive benefit of the value-added service business of power grid enterprise.

In this paper, grey comprehensive evaluation of "e-charging" is performed using the gray whitening weight function constructed. The specific steps are:

\section{Weight Calculation}

Using the AHP method to calculate the weight of each indicator, we can get the weight $w=(0.16,0.11,0.13,0.18,0.07,0.05,0.15,0.15)$.

\section{Expert Assessment}

Five experts are invited to evaluate the observed value of the "e-charging" comprehensive benefit indicator. The score table is shown in Table 2 .

Table 2 “e-charging”Comprehensive Benefit Expert Score Table

\begin{tabular}{|c|c|c|c|c|c|}
\hline Indicator & Expert 1 & Expert 2 & Expert 3 & Expert 4 & Expert 5 \\
\hline $\mathrm{A}_{1}$ & 80 & 78 & 75 & 81 & 79 \\
\hline $\mathrm{A}_{2}$ & 74 & 79 & 82 & 72 & 80 \\
\hline $\mathrm{A}_{3}$ & 85 & 88 & 82 & 91 & 86 \\
\hline $\mathrm{B}_{1}$ & 74 & 83 & 80 & 88 & 70 \\
\hline $\mathrm{B}_{2}$ & 72 & 86 & 84 & 90 & 81 \\
\hline $\mathrm{B}_{3}$ & 88 & 86 & 79 & 80 & 90 \\
\hline $\mathrm{C}_{1}$ & 88 & 94 & 85 & 91 & 89 \\
\hline $\mathrm{C}_{2}$ & 92 & 91 & 95 & 88 & 90 \\
\hline
\end{tabular}

\section{Determine Evaluation Gray Class and Whitening Weight Functions}

The gray class is divided into four categories, which are excellent, good, medium, and poor. The range of scores for the four gray classes is shown in Table 3.The upper limit measurement whitening weight function is used to calculate.

Table 3 The range of scores for the four gray classes

\begin{tabular}{|c|c|c|c|c|}
\hline Gray class & Excellent & Good & Medium & Poor \\
\hline Range of scores & {$[85,100]$} & {$[75,85)$} & {$[60,75)$} & {$[0,60)$} \\
\hline Grey number & {$[85,100,-,-]$} & {$[75,85,-,-]$} & {$[60,75,-,-]$} & {$[0,60,-,-]$} \\
\hline
\end{tabular}

Suppose that when the scores take the boundary values of 60,75 , and 85 , the whitening weight function value is $1 / 2$ of the adjacent score. Namely: when the score $=85$, the whitening weight function for the gray class "excellent" is $1 / 30$; for the score $=75$, the whitening weight function value for the grey class "medium" is $1 / 20$; when the score $=60$, The whitening weight function of the gray class "good" is $1 / 30$.

\section{Grey Comprehensive Evaluation}

According to the whitening weight function formed in Table 2, the whitening weight function values for the four gray classes are calculated for the eight indicators in the available indicator system by the five experts. And according to Eq.2 to 8, we can calculate the grey evaluation weight of the evaluation indicator for each evaluation grey class. The calculation results are summarized in Table 4. 
Table 4 “e-charging”Grey evaluation weights table

\begin{tabular}{|c|c|c|c|c|}
\hline $\begin{array}{c}\text { Grey evaluation } \\
\text { weights }\end{array}$ & Excellent & Good & Medium & Poor \\
\hline $\mathrm{A}_{1}$ & 0.00 & 1.80 & 0.00 & 0.00 \\
\hline $\mathrm{A}_{2}$ & 0.00 & 1.60 & 1.73 & 0.00 \\
\hline $\mathrm{A}_{3}$ & 0.67 & 0.70 & 0.00 & 0.00 \\
\hline $\mathrm{B}_{1}$ & 0.20 & 1.30 & 1.60 & 0.00 \\
\hline $\mathrm{B}_{2}$ & 0.40 & 1.50 & 0.80 & 0.00 \\
\hline $\mathrm{B}_{3}$ & 0.60 & 0.90 & 0.00 & 0.00 \\
\hline $\mathrm{C}_{1}$ & 1.47 & 0.00 & 0.00 & 0.00 \\
\hline $\mathrm{C}_{2}$ & 2.07 & 0.00 & 0.00 & 0.00 \\
\hline
\end{tabular}

According to Eq.9, the weights of each indicator and the grey weights are synthesized and calculated, and the comprehensive clustering results of the four gray classes of the "e-charging" comprehensive benefit of the grid company can be obtained. The calculation results are summarized in Table 5.

Table 5 Result of gray comprehensive evaluation

\begin{tabular}{|c|c|c|c|c|c|}
\hline Evaluation grade & Excellent & Good & Medium & Medium & $\begin{array}{c}\text { Evaluation } \\
\text { conclusion }\end{array}$ \\
\hline $\begin{array}{c}\text { "E-charging"comprehensive } \\
\text { benefit }\end{array}$ & 0.41 & 0.42 & 0.17 & 0.00 & Good \\
\hline
\end{tabular}

The evaluation results show that the "e-charging" has a good comprehensive benefit, and it is specifically represented by good economic benefits, good social benefits and excellent environmental benefits, and it is worthy of promotion.

\section{Conclution}

The paper constructs a comprehensive benefit evaluation index system for the characteristics of value-added services in grid enterprises which is based on three aspects of economic benefits, social benefits and environmental benefits. At the same time, the grey whitening weight function is applied to the study of comprehensive benefit evaluation, which provides a basis for enterprises to improve or optimize existing businesses and provides a reference for similar value-added services business benefit evaluation.

\section{Acknowledgements}

Technology project support by State Grid: Research on value-added service innovation and application technology of power grid enterprises based on full business data. 


\section{Reference}

[1] Lu Haiming, Liu Xiangdong. Research and application of power customer segmentation and value-added service system [J]. North China Electric Power, 2016 (10): 8-13.

[2] Song Caihua. Application of comprehensive value evaluation in customer segmentation of electric power enterprises [J]. Electronic design engineering, 2014, 22(12): 111-116.

[3] William G.Zikmund. Customer Ralationship Management: Integrating Marketing Strategy and Information Technology[M]. 2005: 28.

[4] Liu Xiaoxiao, Tong Yang. Design of multiplex power market electricity value-added service system [J]. Hunan Electric Power, 2013, 33(4): 31-33.

[5] Liu Lin, Wang Xue. Research on the evaluation of smart grid value-added service based on Enterprise Ecology [J]. Journal of North China Electric Power University, 2014, (02): 26-31.

[6] Chen Shoujun, Wei Yanan. Comprehensive benefit evaluation of smart grid based on evidence integration soft set [J]. East China electric power, 2013, 41(12): 2590-2594.

[7] Onen Ahmet. Economic Evaluation of Distribution System Smart Grid Investments[J]. Electric Power Componentd and Systems, 2015, 43(2): 224-233.

[8] Zhao Liang, Li Lili. Evaluation index system and calculation method of smart grid suitable for China's national conditions [J]. Power grid technology, 2015, (12): 3520-3528.

[9] Koji, T. Integrated Smart Grid Evaluation System[J]. Toshiba Leading Innovation, 2011, 66(12): 24-27.

[10]Zhang Jian, Pu Tianjiao. Comprehensive evaluation index system for demonstration project of smart grid [J]. Power grid technology, 2011, 35(6): 5-10. 